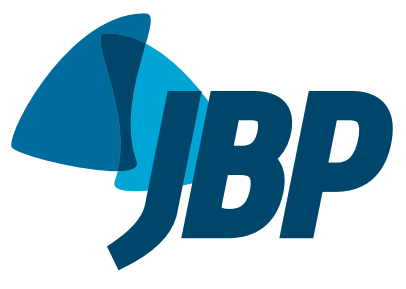

\title{
Pseudomonas aeruginosacolonization in the upper and lower airways of a child with cystic fibrosis: a father's meticulous approach to successful eradication
}

\author{
Jochen Georg Mainz ${ }^{1,2, a}$, Michael Baier ${ }^{3, b}$, Anke Jaudszus ${ }^{1,2, c}$, Harold Tabori²,d, \\ José Dirceu Ribeiro ${ }^{4, e}$, Michael Lorenz ${ }^{1, f}$
}

\section{TO THE EDITOR:}

In cystic fibrosis (CF), an inherited disease, progressive lung destruction, triggered by chronic pulmonary infection with opportunistic pathogens, such as Pseudomonas aeruginosa, is the leading cause of premature death. Consequently, there is a strong incentive for early detection and eradication of $P$. aeruginosa infection. Monitoring lower airway (LAW) colonization and using targeted therapy is the current international standard, which is considered to provide better chances to eradicate the pathogen before it changes to a mucoid phenotype and forms biofilms, which then makes eradication nearly impossible. However, eradication of a novel $P$. aeruginosa colonization is challenging, and success rates vary widely $(60-90 \%)^{(1-3)}$ in divergent protocols consisting of the sole use of inhaled antibiotics, such as tobramycin or colomycin, for periods of about one to six months, or combining oral ciprofloxacin with intravenous antibiotics.

In the last years, the importance of the upper airways (UAW) and the paranasal sinuses for eradication success has been recognized because they are sites for pathogen acquisition, pathogen persistence, LAW contamination, and cross-infection. ${ }^{(4-7)}$ In this context, we implemented routine UAW monitoring by nasal lavage performed every three months as a standard procedure at the CF Center in the University of Jena, in the city of Jena, Germany. Thereby, we previously identified CF patients with primary isolated sinonasal $P$. aeruginosa colonization and we found that identical $P$. aeruginosa strains persisted in the UAW of CF patients after lung transplantation, initially pseudomonas-free transplanted lungs having been colonized with those strains. ${ }^{(8)}$ In addition, we proved that $96 \%$ of the detected $P$. aeruginosa strains were genetically identical in both UAW and LAW of CF patients chronically colonized with that pathogen, which underscores the "united airway" concept. ${ }^{(8)}$

Here, we demonstrate that periodic and close monitoring of LAW and UAW colonization and the use of an aggressive treatment regimen resulted in successful and longstanding eradication of $P$. aeruginosa in a 13-year-old male patient with CF (homozygous F508del genotype). In November of 2011, when the patient was 6 years old, his first $P$. aeruginosa colonization was identified by means of a routine deep throat swab. Subsequently, in accordance with our standard protocol for intermittent colonization, the patient was monthly monitored for UAW colonization (nasal lavage with $10 \mathrm{~mL}$ of isotonic saline per nostril) and LAW colonization (deep throat swabs or sputum collection). ${ }^{(8)}$ After detection of the first $P$. aeruginosa colonization, the father of the patient meticulously documented all culture results and treatments and demanded more efficient therapeutic approaches at every new isolation of the pathogen. Knowing the common standards of therapy, we decided to gradually adapt our approach towards an aggressive eradication regimen with oral or intravenous antibiotics together with inhaled antibiotic therapy. The standard procedure with oral ciprofloxacin and inhaled colistin failed to eradicate $P$. aeruginosa from the LAW (Figure 1), and, five months later, the pathogen was detected in the UAW and LAW. Therefore, we extended the therapy by including quarterly i.v. cycles with ceftazidime and tobramycin and permanent treatment with oral azithromycin. The patients also received colistin by nasal nebulization via a pulsating aerosol nebulizer (PARI SINUS ${ }^{\text {TM}}$; PARI GmbH, Starnberg, Germany). ${ }^{(9)}$ This approach was proven to deliver aerosols into the paranasal sinuses by a superimposed vibration of $43 \mathrm{~Hz}$, applied during periods of breath holding. In contrast, conventional nebulization through the nose was shown not to deliver relevant amounts of nebulized drugs to the sinuses. ${ }^{(10)}$ With the continued intermittent detection of $P$. aeruginosa in both airway compartments, an off-label eradication regimen, consisting of simultaneous inhaled aztreonam for the UAW and LAW, alternated monthly with inhaled tobramycin, was implemented. Subsequently, after nine positive $P$. aeruginosa cultures from the LAW and three from the UAW, $P$. aeruginosa was eradicated in January of 2013, as confirmed by negative cultures from UAW and LAW samples collected every month thereafter. In addition, IgG antibody titers related to the pathogen (alkaline protease, elastase, and exotoxin A) remained negative, as they had been before and during the detection of $P$. aeruginosa in UAW and LAW, in control samples routinely taken every year. At this writing, the patient has remained free of the colonization for more than 72 months, which allowed the therapeutic burden to be reduced to a minimum.

Our experience has shown that additional routine sampling of UAW can reveal early sinonasal colonization, 

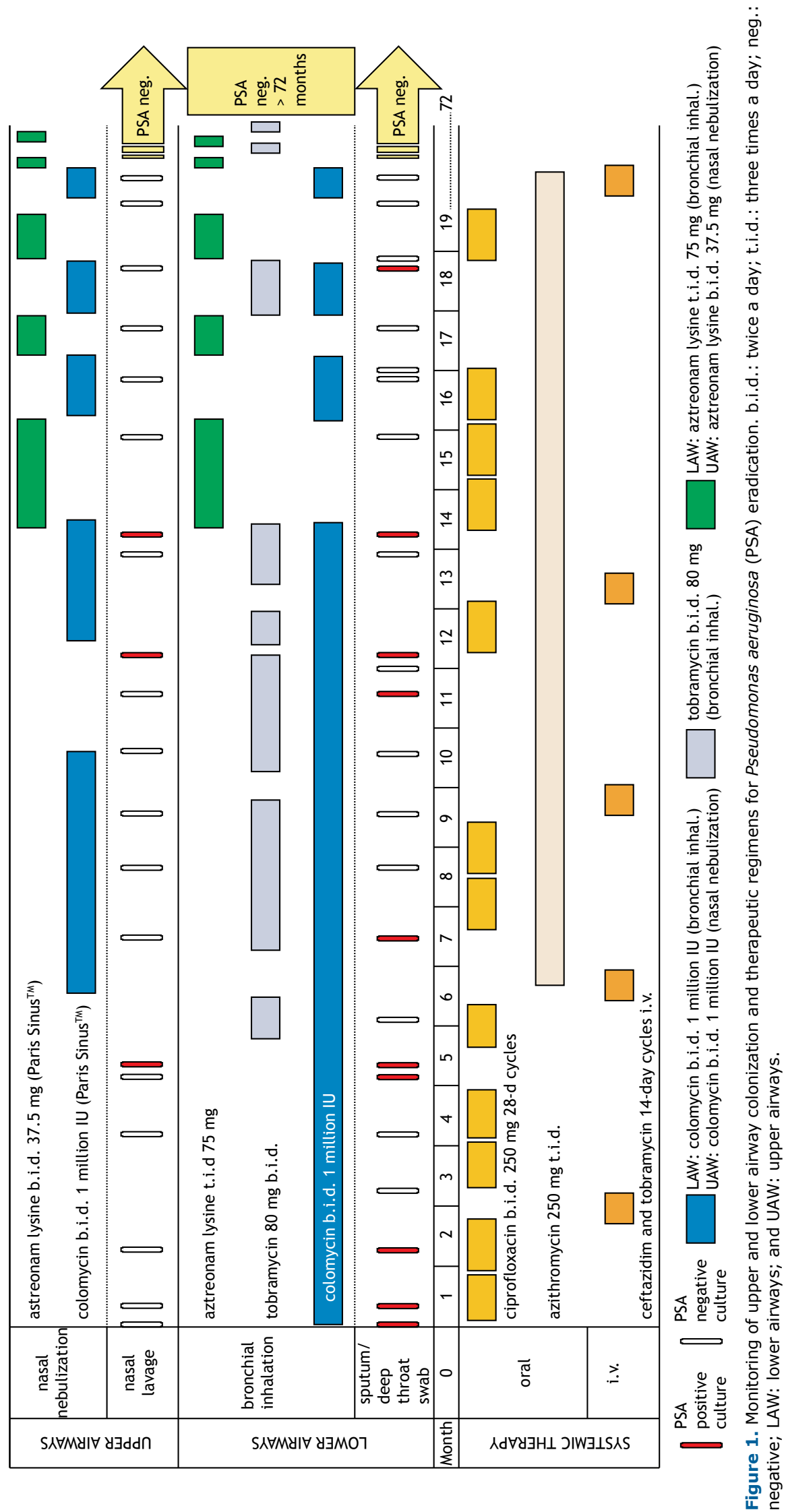
which, if unrecognized, most probably would have prevented the successful eradication of the pathogen. Monthly sampling detected intermittent colonization and later confirmed complete elimination of $P$. aeruginosa. Neither would have been possible if we had followed international standards for CF care, which only recommend LAW sampling 2-4 times/year. The use of inhaled antibiotics alone is likely to fail UAW treatment. This corroborates the findings of a group of authors ${ }^{(5)}$ who showed that if $P$. aeruginosa is not eradicated from the entire airway system, it can undergo evolution and diversification processes in order to adapt to the immune system of the host in the paranasal sinuses. If the paranasal sinuses are not considered as a possible reservoir for $P$. aeruginosa, after the cessation of an inhaled therapy alone, the pathogen may descend to the lungs and cause a new pulmonary colonization that can be even more virulent.

No side effects were reported during the off-label eradication regimen. However, the daily burden of treatment widely exceeded the regular number of inhalations in CF patients. Nevertheless, our patient has been free of colonization for 6 years, during which the therapeutic burden could be reduced to a minimum. We show here that the eradication of persistent $P$. aeruginosa is possible with a consistent therapeutic regimen that, in this case, was strongly promoted by the insistence of the family. This may have contributed to a relevantly improved prognosis of the pulmonary function and, thereby, life expectancy of the patient. Based on our experience, we recommend repeated microbiological assessment of both UAW and LAW, especially in patients with intermittent $P$. aeruginosa colonization. ${ }^{(6)}$ Consequently, such an approach should also be included in future studies on $P$. aeruginosa eradication in CF patients.

\section{ACKNOWLEDGMENTS}

The whole process was closely administered and monitored by the father of the patient. We want to thank him and the entire family for their commitment. Mr. Mainz received fees for lectures from PARI GmbH and Vertex $\mathrm{GmbH}$.

\section{REFERENCES}

1. Blanchard AC, Horton E, Stanojevic S, Taylor L, Waters V, Ratjen F. Effectiveness of a stepwise Pseudomonas aeruginosa eradication protocol in children with cystic fibrosis. J Cyst Fibros. 2017;16(3):395 400. https://doi.org/10.1016/j.jcf.2017.01.007

2. Ratjen F, Munck $A$, Kho P, Angyalosi G. Treatment of early Pseudomonas aeruginosa infection in patients with cystic fibrosis: the ELITE trial. Thorax. 2010;65(4):286-91. https://doi.org/10.1136/ thx.2009.121657

3. Proesmans $M$, Vermeulen F, Boulanger L, Verhaegen J, De Boeck K. Comparison of two treatment regimens for eradication of Pseudomonas aeruginosa infection in children with cystic fibrosis. J Cyst Fibros. 2013;12(1):29-34. https://doi.org/10.1016/j. jcf.2012.06.001

4. Mainz JG, Hentschel J, Schien C, Cramer N, Pfister W, Beck JF, et al. Sinonasal persistence of Pseudomonas aeruginosa after lung transplantation. J Cyst Fibros. 2012;11(2):158-61. https://doi org/10.1016/j.jcf.2011.10.009

5. Hansen SK, Rau MH, Johansen HK, Ciofu O, Jelsbak L, Yang L, et al. Evolution and diversification of Pseudomonas aeruginosa in the paranasal sinuses of cystic fibrosis children have implications for chronic lung infection. ISME J. 2012;6(1):31-45. https://doi. org/10.1038/ismej.2011.83
6. Aanæs K. Bacterial sinusitis can be a focus for initial lung colonisation and chronic lung infection in patients with cystic fibrosis. J Cyst Fibros. 2013;12 Suppl 2:S1-20. https://doi.org/10.1016/S15691993(13)00150-1

7. Folkesson A, Jelsbak L, Yang L, Johansen HK, Ciofu O, Høiby N, et al Adaptation of Pseudomonas aeruginosa to the cystic fibrosis airway: an evolutionary perspective. Nat Rev Microbiol. 2012;10(12):841-51. https://doi.org/10.1038/nrmicro2907

8. Mainz JG, Naehrlich L, Schien M, Kading M, Schiller I, Mayr S, et al. Concordant genotype of upper and lower airways $P$ aeruginosa and $S$ aureus isolates in cystic fibrosis. Thorax. 2009;64(6):535-40. https:// doi.org/10.1136/thx.2008.104711

9. Mainz JG, Schädlich K, Schien C, Michl R, Schelhorn-Neise P, Koitschev $A$, et al. Sinonasal inhalation of tobramycin vibrating aerosol in cystic fibrosis patients with upper airway Pseudomonas aeruginosa colonization: results of a randomized, double-blind, placebo-controlled pilot study. Drug Des Devel Ther. 2014;8:209-17. https://doi.org/10.2147/DDDT.S54064

10. Moller W, Saba GK, Haussinger K, Becker S, Keller M, Schuschnig U. Nasally inhaled pulsating aerosols: lung, sinus and nose deposition. Rhinology. 2011;49(3):286-91. https://doi.org/10.1164/ajrccmconference.2011.183.1_MeetingAbstracts.A4432 